\title{
Essai de calcul des hydrogrammes à partir des pluies: Cas de la Corrèze à Brive
}

\author{
An attempt to calculate a hydrograph \\ from rainfall data : \\ Case of the Corrèze at Brive
}

PAR J. L. LACROIX

INGÉNIEUR D'ARRONDISSEMENT DES PONTS ET CHAUSSÉES

\begin{abstract}
Calcul des débits à partir des pluies réelles par une méthode utilisant les données de toutes les crues, de la Corrèze à Brive, observées depuis janvier 1957 et, notamment, la grande crue d'octobre 1960; les transformations tiennent compte automatiquement :

- des déficits d'écoulement, dont le paramètre principal serait une sorte de débit de base du cours d'eau (écoulement hypodermique et souterrain);

- de l'étalement dans le temps, par la variation des temps de propagation en fonction des débits.

Ajustement favorable de la méthode aux données pour 10 crues différentes couvrant au total 80 jours.
\end{abstract}

Calculation of flow rates from observed rainfall by a method using all the flood data observed on the Corrèze at Brive since January 1957 , especially the major flood that occured in October 1960. The transformations automatically allow for the following:

i) The "flow deficit," the main parameter of which appears to be a sort of base flow of the river (hypodermic and underground flow); ii) The spreading out of these conditions in time, owing to the variation of the propagation times with flow rates.

The method was satisfactorily adjusted to the data for 10 different floods covering a total period of 80 days.

\section{NOTE PRELIMINAIRE}

Dans une précédente étude sur les crues de l'Isle, de la Vézère et de la Corrèze, nous avions appliqué la méthode de l'hydrogramme unitaire avec des compléments relatifs au calcul de l'écoulement hypodermique et à la correspondance entre les pluies réelles et les pluies nettes.

Mais cette étude, à défaut de renseignements détaillés antérieurs à 1957 , ne portait que sur des crues faibles ou moyennes.

La grande crue d'octobre 1960 sur la Corrèze et la Vézère nous a apporté, de ce fait, des renseignements de la plus grande importance; il est apparu, en particulier, que, dans le bassin de la Corrèze, l'augmentation des débits correspon- 
dait à un fort raccourcissement des temps de propagation.

Cette dernière constatation nous a conduit à remplacer la méthode de l'hydrogramme unitaire par un procédé plus nuancé.
Dans le cas de la Corrèze à Brive, qui fait l'objet du présent exposé, nous avons pu, ainsi, obtenir une correspondance acceptable pour la crue d'octobre et améliorer, en les reprenant, nos études antérieures.

\section{DONNEES GENÉRALES \\ SUR LE BASSIN DE LA CORREZE A BRIVE}

Ce bassin est représenté par la carte figure 1; nous $y$ avons figuré les cours d'eau, les stations pluviométriques et le découpage approximatif admis dans notre étude entre :

- un bassin amont (d'environ $270 \mathrm{~km}^{2}$ );

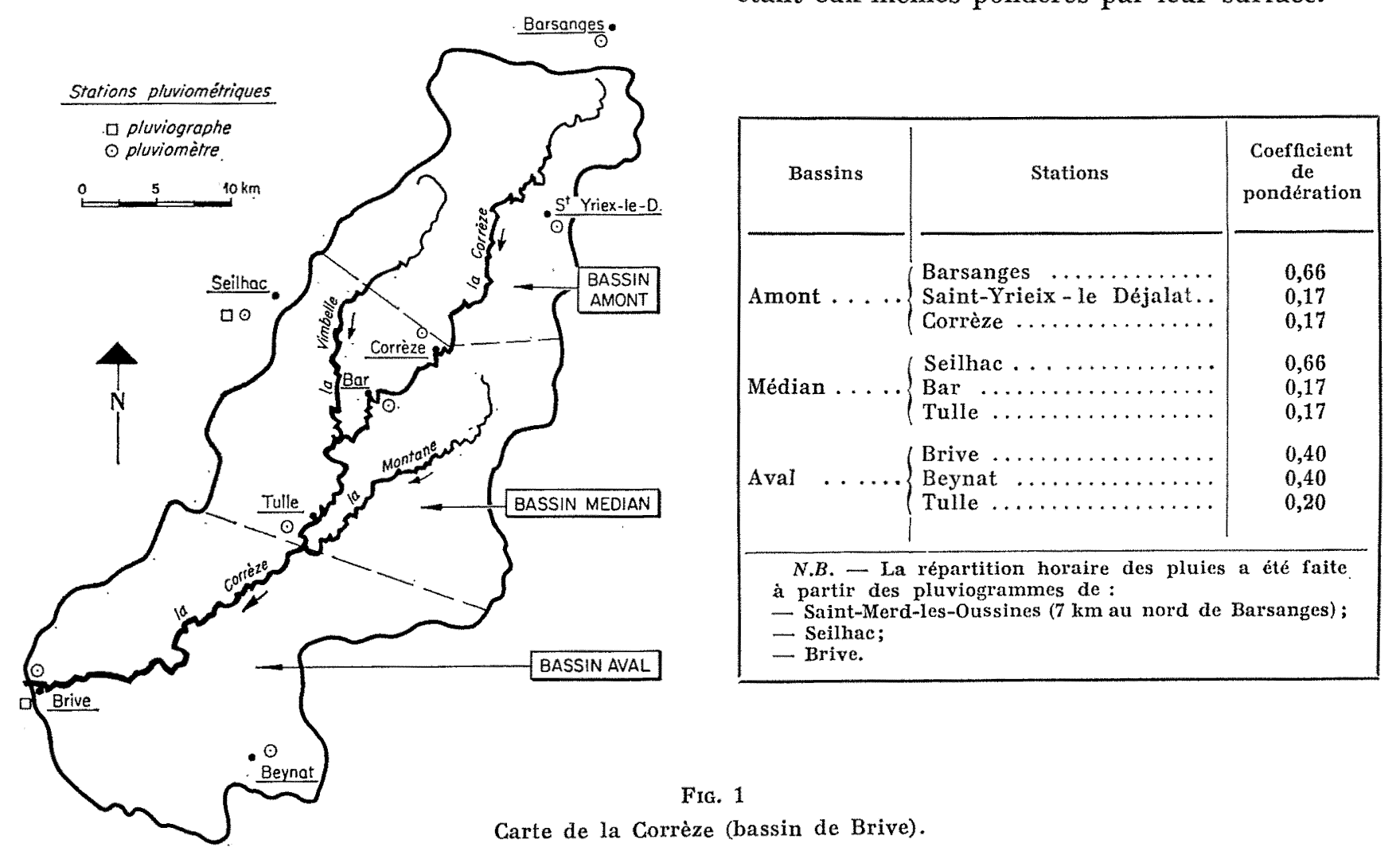

- un bassin médian (d'environ $340 \mathrm{~km}^{2}$ );

- un bassin aval (d'environ $340 \mathrm{~km}^{2}$ ).

Nous avons mentionné, en outre, sur le tableau ci-dessous, le coefficient de pondération que nous avons affecté à chaque station, les bassins étant eux-mêmes pondérés par leur surface.

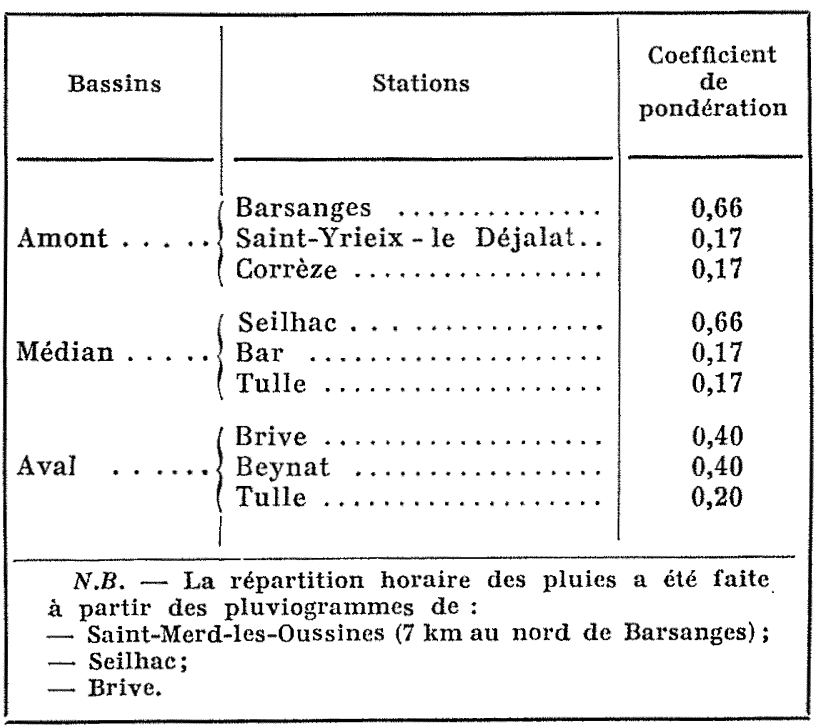

Carte de la Corrèze (bassin de Brive). 


\section{EXPOSE DE LA MÉTHODE SUIVIE}

Cette méthode peut être représentée par le schéma suivant :

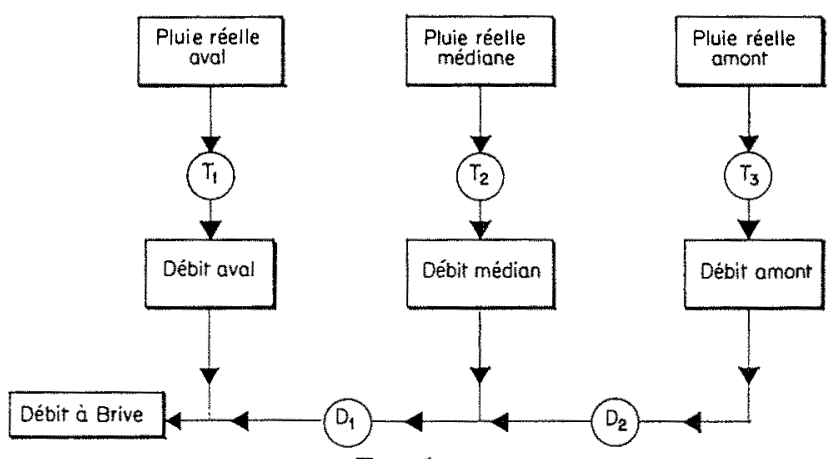

FIG. $1 a$

$\mathrm{T}_{1}, \quad \mathrm{~T}_{2}, \quad \mathrm{~T}_{3}$ représentant des transformations pluie-débit que nous allons préciser, et $D_{1}, D_{2}$ des décalages de propagation dans le cours d'eau principal, décalages qui sont donnés par la figure 2 (nous avons la même loi pour $D_{1}$ et $\mathrm{D}_{2}$ ).

Les transformations $\mathrm{T}$ sont constituées comme suit :

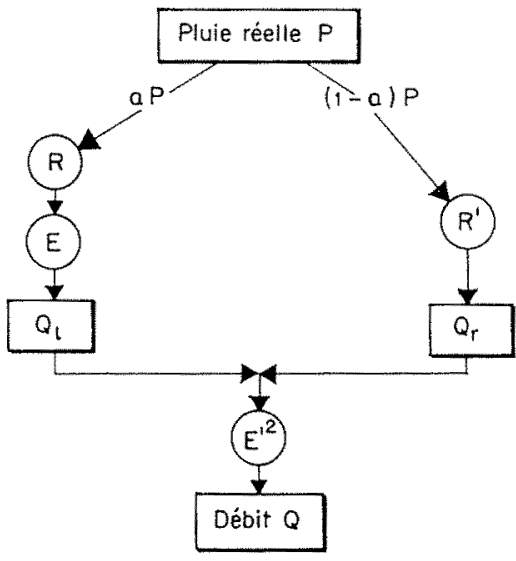

$$
\begin{aligned}
& \text { Valeurs de a : } \\
& \text { bassin amont.... } 0,78 \\
& \begin{array}{l}
\text { bassins median } \\
\text { et aval }
\end{array} 0,72
\end{aligned}
$$

Fix. $1 b$

où :

- $\mathrm{R}, \mathrm{R}^{\prime}$ sont des transformations de réduction permettant de passer des pluies réelles aux pluies nettes.

- E, E' sont des transformations d'étalement dans le temps qui assurent le passage des hyétogrammes aux hydrogrammes.
- $\mathrm{Q}_{l}$ et $\mathrm{Q}_{r}$ sont les deux composantes du débit, la première $Q_{l}$ correspondant à un écoulement relativement lent (plusieurs jours pour les crues faibles ou moyennes), la deuxième, $Q_{r}$, correspondant à un écoulement relativement rapide (de l'ordre de 5 à 10 heures pour les mêmes crues).

Définition des transformations $R, R^{\prime}, E, E^{\prime}$

$R, \mathrm{R}^{\prime}, \mathrm{E}, \mathrm{E}^{\prime}$ constituent les transformations spécifiques de la correspondance pluie-débit.

Leurs caractéristiques et le choix des paramètres qui y sont utilisés résultent des très nombreux essais que nous avons effectués pendant deux ans pour l'interprétation des diverses crues.

\section{a) Transformations de réduction $\mathrm{R}$ et $\mathrm{R}^{\prime}$ :}

Les transformations de réduction $\mathrm{R}$ et $\mathrm{R}^{\prime}$ utilisent comme paramètres le débit $Q_{l}$ et la pluie réelle $\mathrm{P}$.

N'ayant pas d'éléments suffisamment sûrs pour traiter séparément les trois bassins, nous avons admis les mêmes fonctions dans les trois

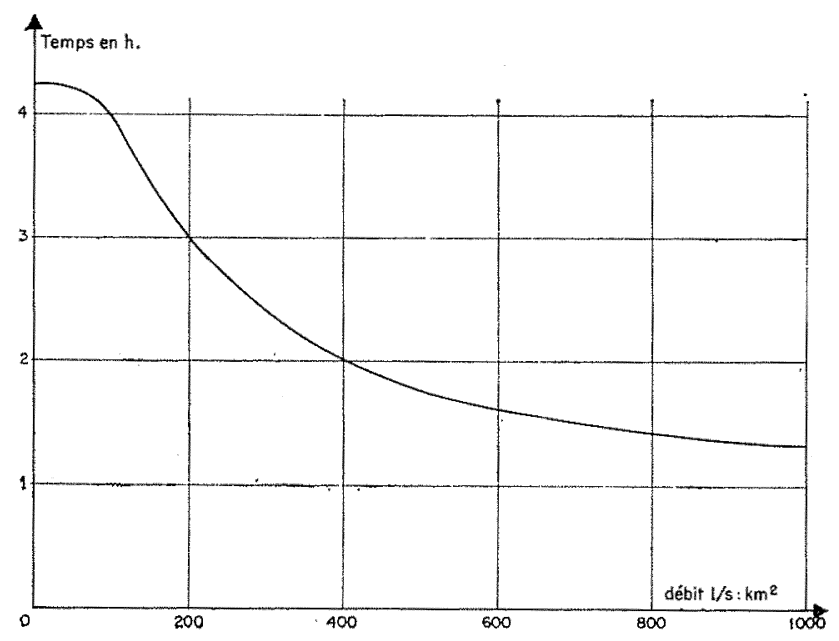

FIG. 2

Décalages de propagation le long du cours d'eau principal. 
cas; ces fonctions sont représentées par les figures 3 et 4 .

Ces fonctions ne semblent, toutefois, valables qu'en automne et en hiver (d'octobre à mars); au printemps et en été, les quelques exemples de crue que nous avons pu examiner nous conduisent à proposer (sous réserve d'une plus longue expérience) un terme correctif qui se présente sous forme d'un coefficient $c$ à appliquer aux pertes $1-R$ intervenant dans le calcul de $\mathrm{Q}_{l}$ (voir également figure 3 ).

Il est à remarquer que ce coefficient réagissant sur $\mathrm{Q}_{l}$ réagit aussi, de ce fait, sur $\mathrm{Q}_{r}$; d'autre part, la forme de la réduction $\mathrm{R}$ est telle que $c$ n'intervient pratiquement qu'en période sèche (faibles valeurs de $\mathrm{Q}_{l}$ ).

\section{b) Transformations d'étalement $\mathbf{E}$ et $\mathrm{E}^{\prime}$ :}

Ce sont des transformations qui conservent les volumes; d'une façon générale, on peut les schématiser comme suit :

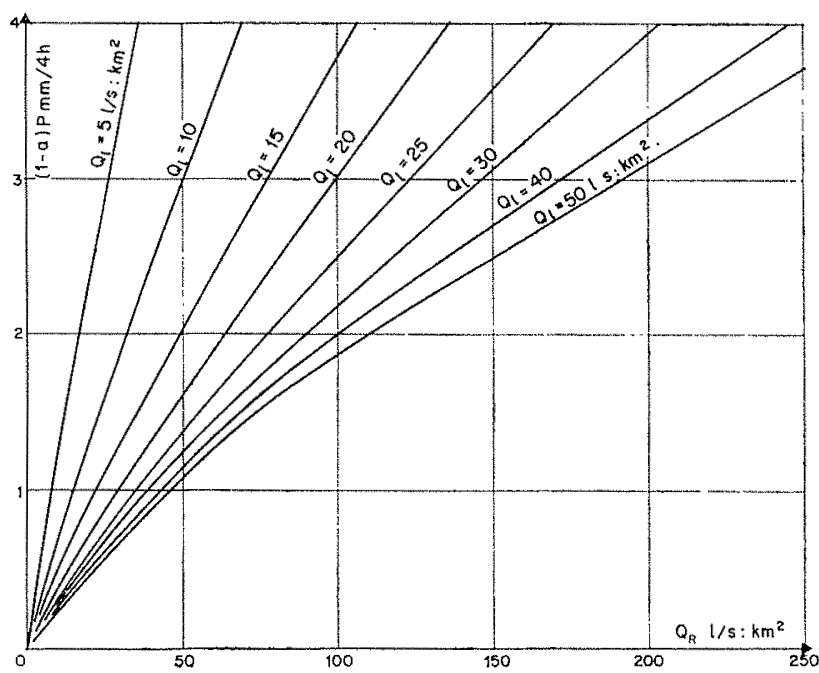

Fig. 4

Transformation de réduction $R^{\prime}$.

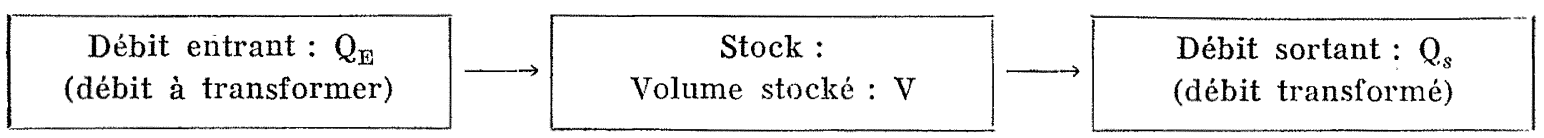

le volume stocké intermédiaire s'annulant pratiquement à la fin de chaque période pluvieuse.

Parmi les diverses transformations de ce genre, nous avons choisi comme outil de calcul celles que nous désignerons par (é) qui correspondent au cas simple où le débit transformé $\mathbf{Q}_{s}$ est uniquement fonction du volume stocké $\mathrm{V}$ :

$$
\mathrm{Q}_{s}=f(\mathrm{~V})
$$

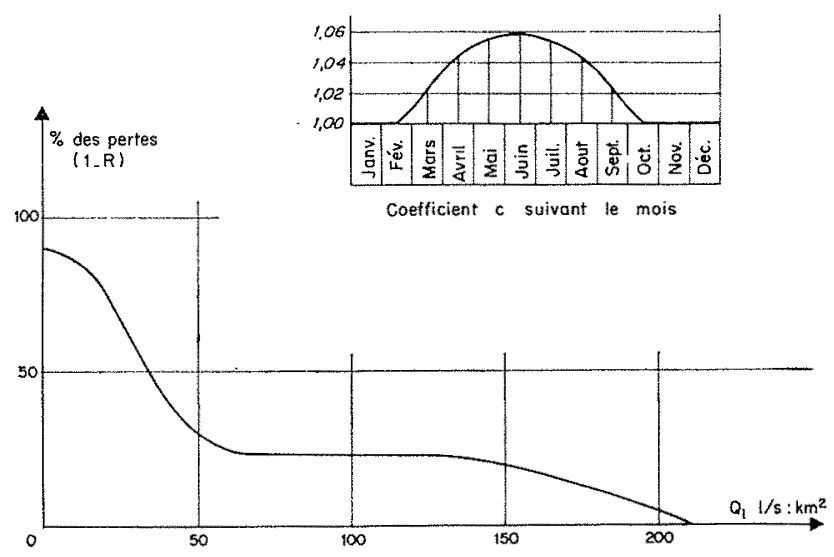

Fig. 3

Transformation de réduction $R$ ou, sous une autre forme :

$$
\frac{d \mathrm{~V}}{d \mathrm{Q}_{s}}=\mathrm{T}\left(\mathrm{Q}_{s}\right)
$$

$\mathrm{T}\left(Q_{s}\right)$ étant une fonction quelconque, homogène à un temps, que nous proposons d'appeler temps caractéristique, car elle représente abstraction faite du débit entrant - la rapidité de vidange du stock pour le débit $Q_{s}$.

Elle s'identifie notamment à la constante de temps des écoulements exponentiels.

La fonction $T\left(Q_{s}\right)$ présente, en outre, l'intérêt d'être facilement lisible sur les hydrogrammes de crue (pente par rapport à l'axe des débits des courbes de tarissement représentées en coordonnées semi-logarithmiques).

En appliquant des transformations (e) (une ou plusieurs à la suite), on obtient des résultats correspondant à des hydrogrammes unitaires variables, modelés en fonction des débits.

Pour plus de précision, nous dirons que :

- le choix de la fonction $\mathrm{T}\left(\mathrm{Q}_{s}\right)$ permet de modeler surtout la partie décroissante des hydrogramnies unitaires.

-- la partie croissante de ces mêmes hydrosrammes (instantanée dans le cas d'une seule transformation (e) pourra être étalée en 


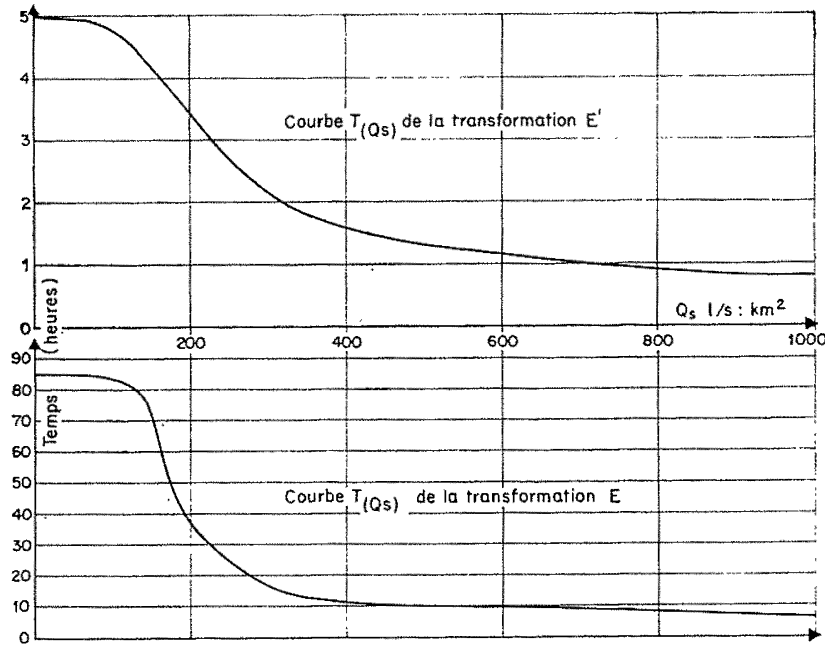

Fig. 5

appliquant successivement deux (ou peutêtre trois) transformations du type $(e)$.

Ainsi, dans le cas de la Corrèze à Brive, nous avons pris pour transformations $E$ et $E^{\prime}$ des transformations du type $(e)$ dont les courbes $\mathrm{T}\left(\mathrm{Q}_{s}\right)$ sont représentées sur la figure 5 .

La transformation $\mathrm{E}$ qui correspond à l'écoulement lent a des temps caractéristiques très supérieurs à ceux de la transformation $\mathrm{E}^{\prime}$.
Quant à la transformation $E^{\prime}$ dont l'application se substitue à celle de l'hydrogramme unitaire, elle est appliquée deux fois $\left(\mathrm{E}^{\prime 2}\right)$ de façon $\grave{a}$ adoucir convenablement le front des ondes de crue; à titre d'exemple, nous avons donné figure 6 les hydrogrammes unitaires instantanés correspondant, pour divers débits, à l'application de $\left(\mathrm{E}^{\prime 2}\right)$; ils sont nettement différents les uns des autres.

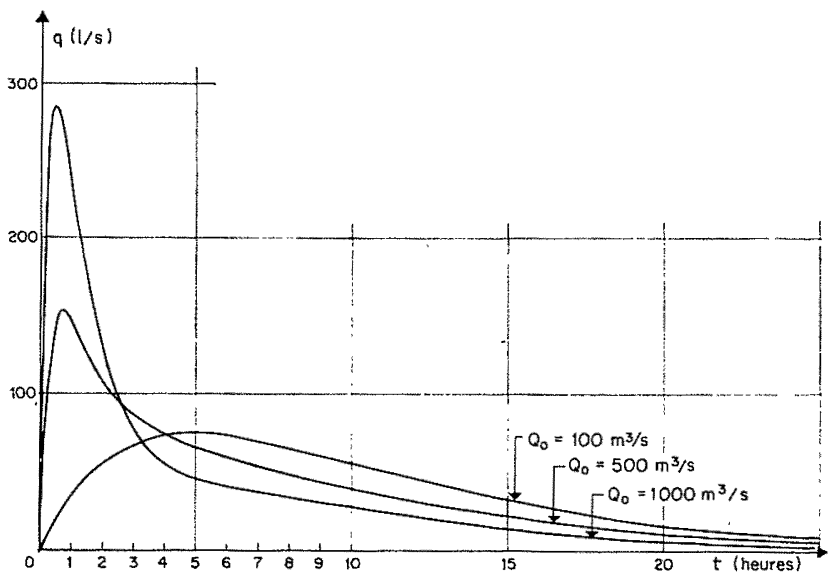

FIG. 6

Exemple d'hydrogrammes unitaires instantanés correspondant à l'application $\mathrm{E}^{\prime 2}$ pour un volume de $1 \mathrm{~m}^{3}$.

N.B. - - Les valeurs de $\mathrm{Q}_{0}$ correspondent à l'ensemble $\mathrm{du}$ bassin $\left(950 \mathrm{~km}^{2}\right)$.

\section{EXECUTION PRATIQUE DES CALCULS}

Les calculs d'application ont été effectués essentiellement par des procédés graphiques (de même que les diverses transformations précitées ont été définies par le tracé de courbes sans aucun souci d'expression analytique).

Pour chaque crue, nous sommes partis de la période sans pluie qui l'a précédée où l'on peut prendre pour chaque bassin (amont, médian, aval) :

$$
\begin{aligned}
& \mathrm{Q}_{r}=0 \\
& \mathrm{Q}_{t}=Q \text { (débit total réel). }
\end{aligned}
$$

Un premier abaque nous a donné, par simple pointé, sur un graphique :

- à partir du débit de pluie et du débit $Q_{l}$ à l'instant $t$ les débits $Q_{l}$ et $Q_{l}+Q_{r}$ à l'instant $t+\Delta t[\Delta t$ étant pris normalement égal à 4 heures et exceptionnellement (grande crue d'octobre) à 1 heure].

Un deuxième abaque nous a donné, également par simple pointé sur un graphique, le résultat de la transformation $\mathrm{E}^{\prime}$, soit :

- à partir du débit transformé à l'instant $t-(\Delta t / 2)$ et du débit à transformer à l'instant $t$ le débit transformé à l'instant

$$
t+(\Delta t / 2)
$$

(avec les mêmes valeurs de $\Delta t$ que pour le premier abaque).

Cette dernière opération a été répétée deux fois $\left(\mathrm{E}^{\prime 2}\right)$.

Les pluies étant données, chaque point de l'hydrogramme d'un bassin (amont, médian, aval) a nécessité un temps de calcul de l'ordre de la minute (trois lectures d'abaque).

Les hydrogrammes de trois bassins ont été ensuite additionnés avec les décalages de propagation par simple report graphique.

En définitive, le tracé des hydrogrammes à partir des pluies est relativement rapide, le plus long n'est certes pas d'appliquer les abaques mais de les tracer... 


\section{RESULTATS OBTENUS}

Les résultats obtenus sont représentés sur le graphique des figures 7 et suivantes.

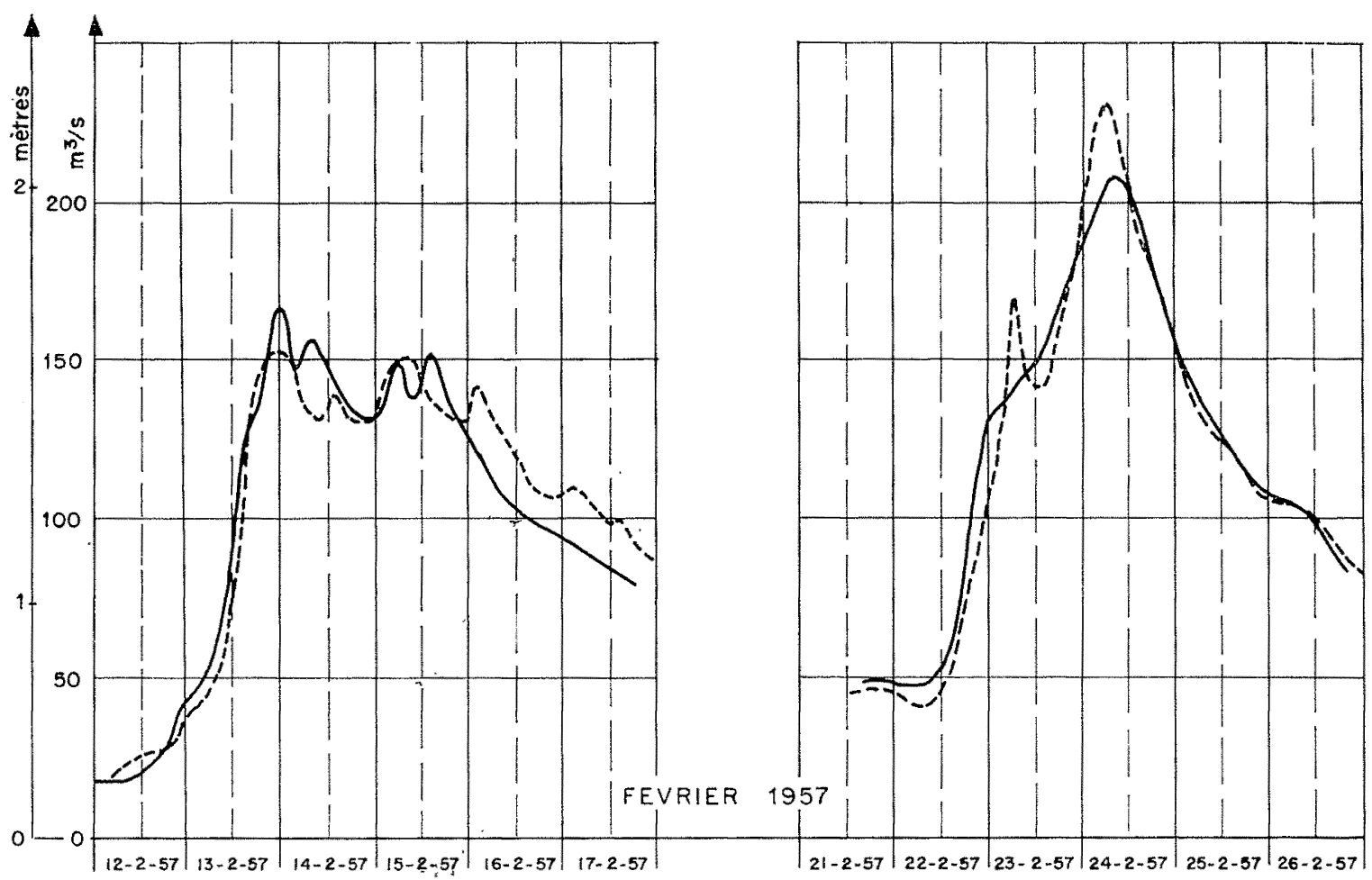

FIG. 7
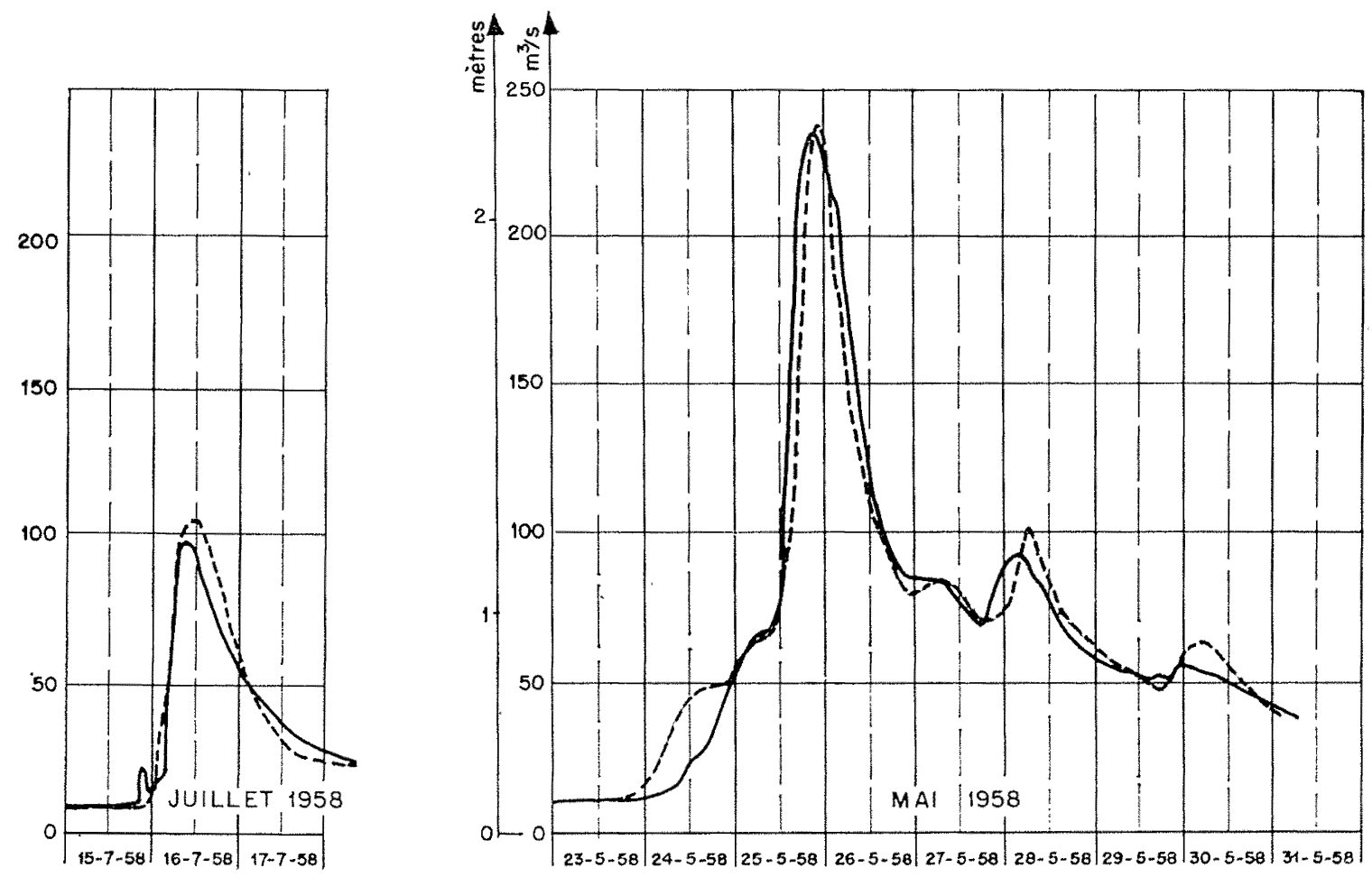

Frg. 8 
Nous avons appliqué systématiquement notre méthode à toutes les crues de quelque importance sur lesquelles nous disposions de renseignements pluviométriques et hydrométriques convenables, c'est-à-dire à partir de janvier 1957.

Les hydrogrammes réels sont représentés par un trait plein et les hydrogrammes calculés par un trait pointillé.
Bien entendu, nous avons choisi les diverses transformations utilisées de façon à obtenir les meilleurs résultats possibles.

Il semble, cependant, que le nombre et la variété des cas où la concordance entre les deux courbes reste assez bonne autorise quelque espoir quant aux prévisions futures.

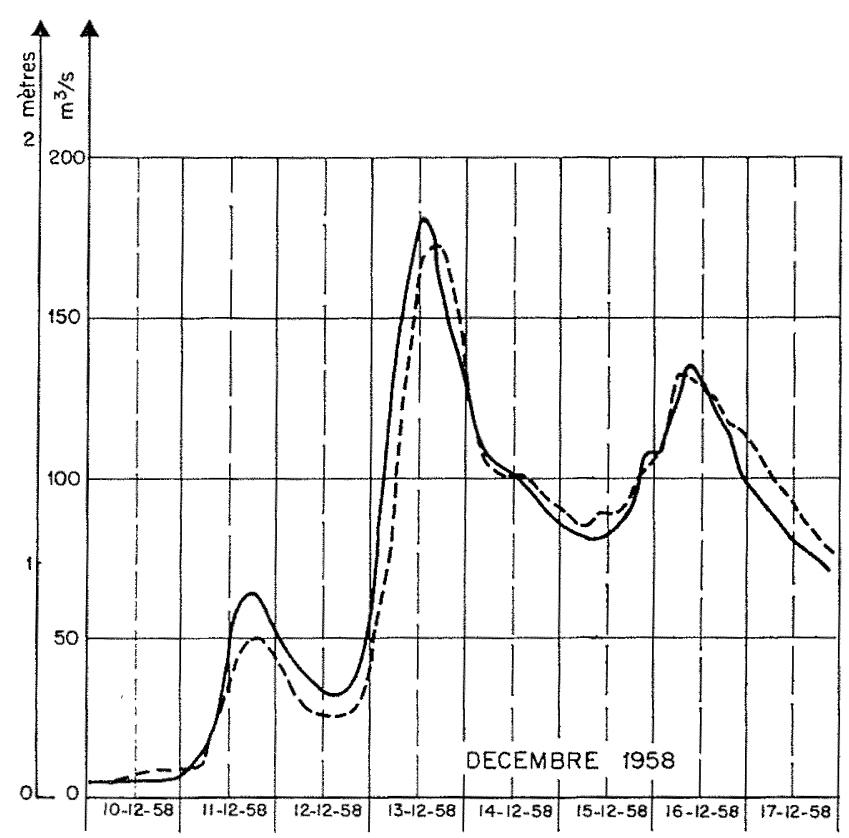

FIG. 9

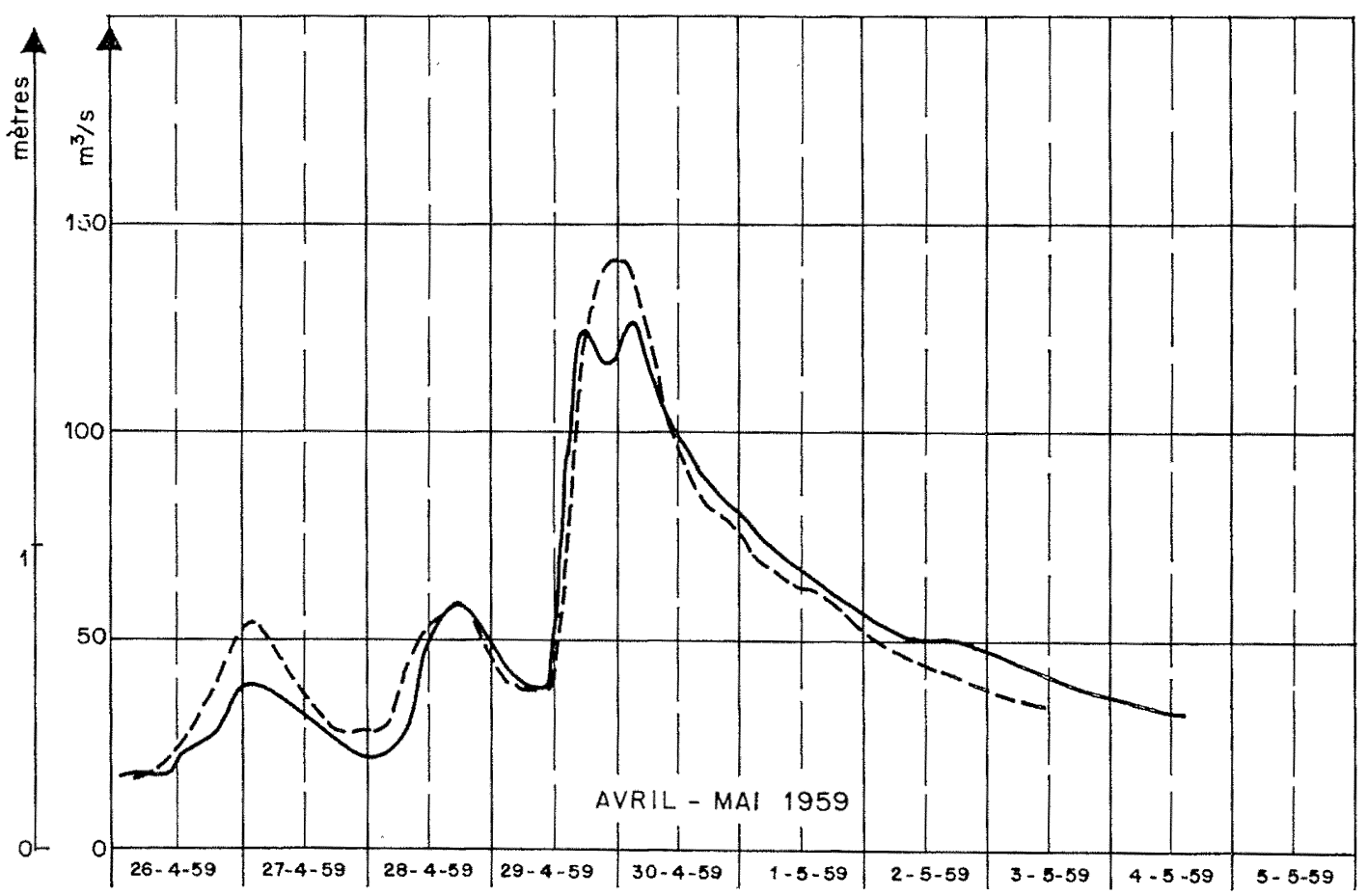

FIG. 10 


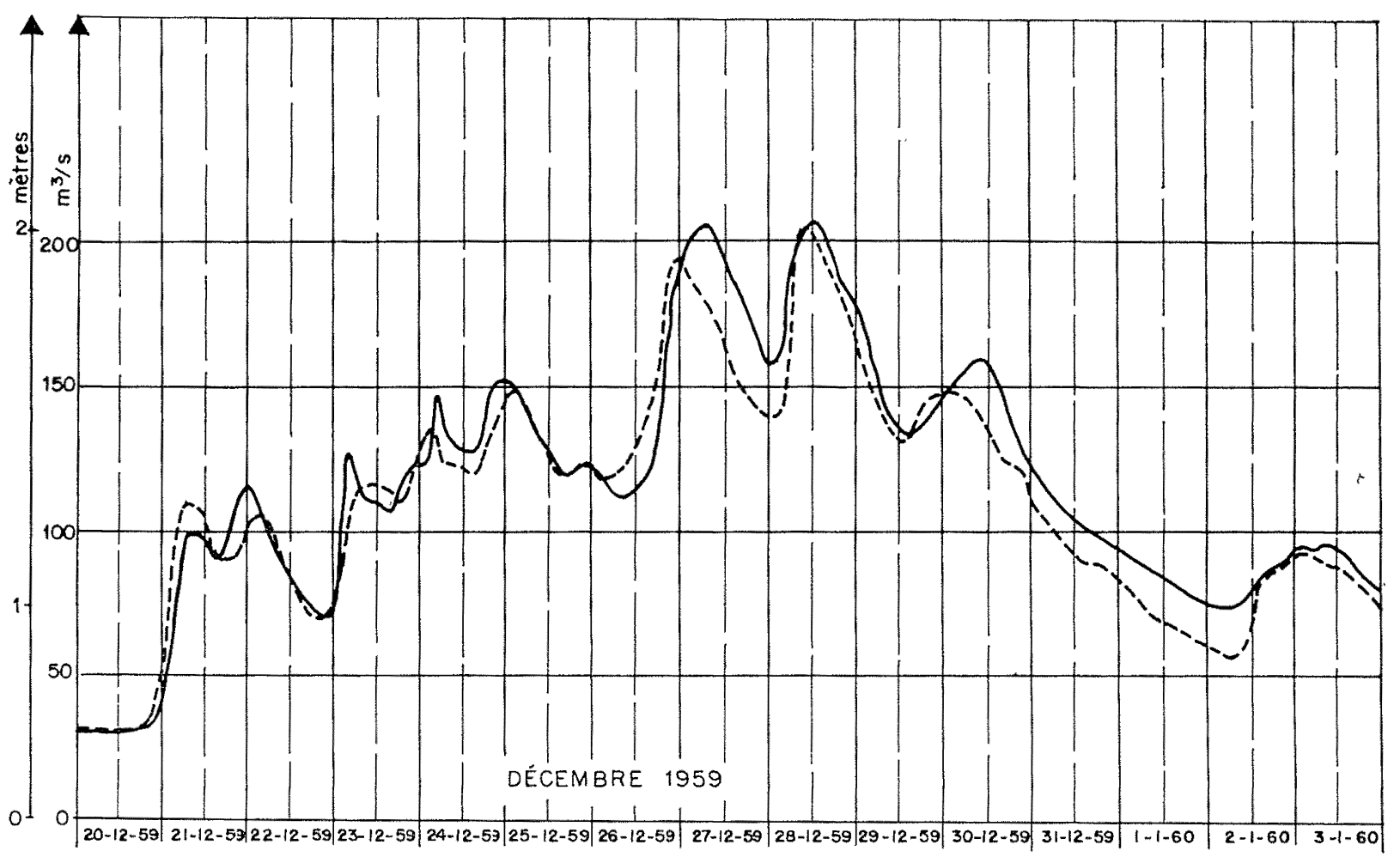

FIG. 11

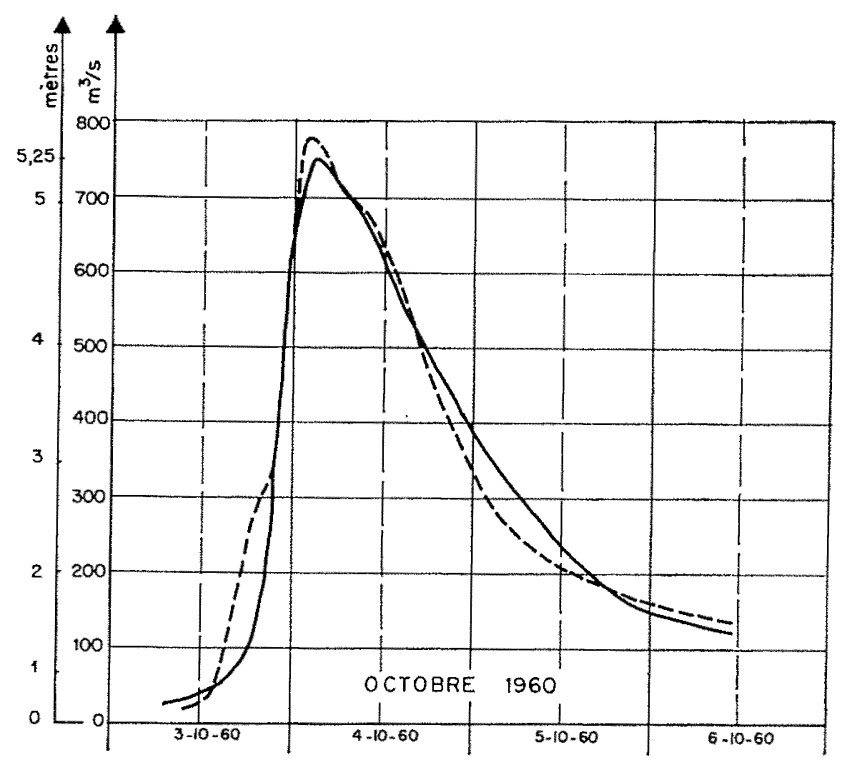

Fig. 12.

Crue exceptionnelle d'octobre 1960.

N.B. - L'échelle des débits est beaucoup plus réduite que dans les autres exemples, étant donné l'importance de la crue. 


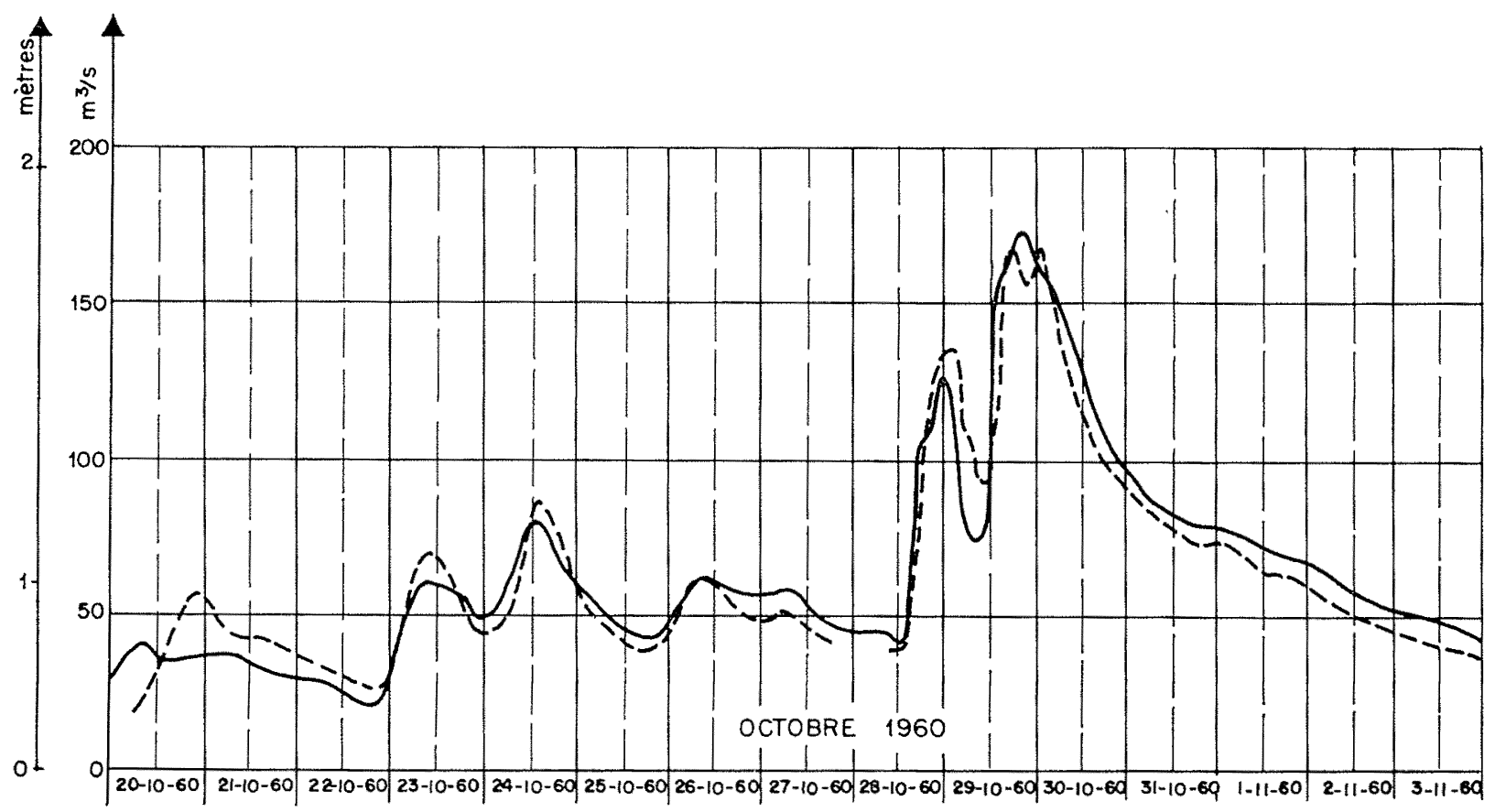

FIG. 13

Ces résultats ont été obtenus par un travail important de documentation et de calculs dont je tiens à remercier :

- le Service hydrométéorologique de l'E.D.F., - le Service d'étude des débits de la $4^{\text {e }}$ circonscription électrique,

- le Centre de Limoges de la Météorologie Nationale,

pour toute la documentation si obligeamment communiquée par ces organismes;

- tous les agents de mon arrondissement (Ingénieurs T.P.E., Adjoint Technique, Dessinateurs) qui ont contribué à cette étude, pour le travail fastidieux qui a été le leur.

\section{I S C U S I O N}

Président : M. de Rouville

M. le Président donne la parole à M. LAcroix, qui félicite M. JACQUET de la clarté de sa synthèse et déclare n'avoir aucune observation à faire, sinon qu'il fat se garder de généraliser son travail, qui est surtout un essai qu'il convient de confronter avec d'autres résultats d'observations.

M. le Président est frappé de la similitude des situations météorologiques qui ont produit les crues des Cévennes en 1958, et celles de la région de Millevaches en 1960. Aurait-on pu prévoir, à les considérer, que les pluies et les crues intéresseraient le versant méditerranéen dans le premier cas, celui de l'Océan dans le second? C'est ce qui paraît fort douteux dans l'état actuel des données et connaissances météorologiques.

FIG. 14

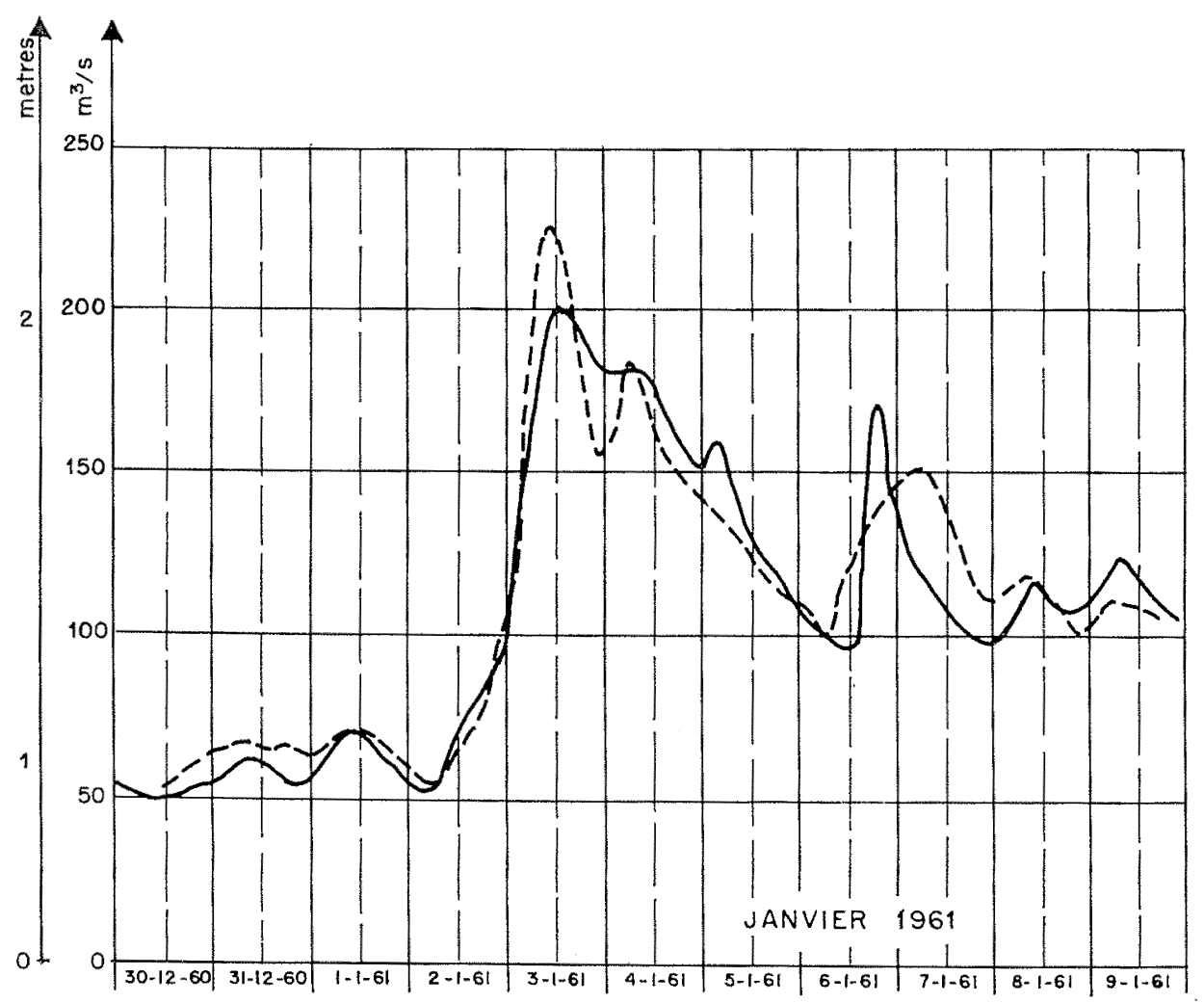

